\title{
Laparoscopic Gastrojejunostomy with Conversion Therapy in Gastric Outlet Obstruction Caused by Incurable Advanced Gastric Cancer
}

\author{
Chuandong Wang ${ }^{1,2}$ \\ Shengtao Lin ${ }^{1,2}$ \\ Xiaojuan Zhang ${ }^{3,4}$ \\ Changshun Yang ${ }^{1,2}$ \\ Weihua $\mathrm{Li}^{1}$
}

'Shengli Clinical Medical College of Fujian Medical University, Fuzhou, 35000I, People's Republic of China; ${ }^{2}$ Department of Surgical Oncology, Fujian Provincial Hospital, Fuzhou, 35000I, People's Republic of China; ${ }^{3}$ Fuzong Clinical Medical College of Fujian Medical University, Fuzhou, 35000I, People's Republic of China; ${ }^{4}$ Department of Radiology, 900th Hospital Logistic Support Forces of PLA, Fuzhou, 35000I, People's Republic of China
Correspondence: Weihua Li

Shengli Clinical Medical College of Fujian Medical University, No. 134, East Street,

Fuzhou, 35000I, People's Republic of China

Tel +86- $|8|-0606 \mid 193$

Fax +86-59I-882I7I50

Email liwh@fjmu.edu.cn
Purpose: The benefits of laparoscopic gastrojejunostomy (LGJ) combined with conversion therapy for malignant gastric outlet obstruction (GOO) caused by incurable advanced gastric cancer (AGC) are unclear. This study aimed to examine the feasibility and efficacy of LGJ followed by enteral nutrition and conversion therapy in malignant GOO.

Patients and Methods: The clinical outcomes for 66 patients with GOO due to incurable AGC were retrospectively evaluated. The patients were classified into multimodal therapy (LGJ, enteral nutrition, and chemotherapy, $n=35)$ and chemotherapy alone $(n=31)$ groups. Conversion surgery was defined as surgery aimed at R0 resection in initially incurable tumours. Results: Compared to the chemotherapy group, multimodal therapy patients had improved oral intake, more chemotherapy cycles, better nutritional indices, less sarcopenia, and improved quality of life (QOL) post-treatment. Conversion surgery was performed in 17 multimodal therapy patients, with no perioperative mortality, and R0 resection achieved in 15 patients $(88.2 \%)$. The median survival time of multimodal therapy patients was 16.7 months, compared to 4.5 months for chemotherapy patients. Multimodal therapy patients with conversion surgery had significantly longer overall survival than those without surgery (44.2 vs 8.5 months, respectively, $\mathrm{P}<0.001$ ). Multivariate analysis identified multimodal therapy and improved or stable QOL as independent prognostic factors.

Conclusion: Multimodal therapy was associated with better nutritional and metabolic status, a safely induced high conversion surgery rate with a high R0 resection rate, and a good prognosis. LGJ with enteral nutrition and conversion therapy may improve long-term survival in obstructive incurable AGC.

Keywords: gastric cancer, gastrojejunostomy, conversion therapy, metabolism, enteral nutrition

\section{Introduction}

Gastric cancer is frequently diagnosed at an incurable advanced stage with dismal prognosis. ${ }^{1}$ The therapeutic treatment for such patients may be palliative chemotherapy. In recent times, growing literature has reported improved life expectancy for those who responded to chemotherapy and underwent surgery aimed at R0 resection, or "conversion surgery". ${ }^{2-5}$ Unfortunately, gastric outlet obstruction (GOO) is the most detrimental feature ofAGC. As a result, patients' nutritional status and metabolic patterns deteriorate, depriving them of the opportunity for conversion therapy. ${ }^{6}$ Thus, eliminating obstruction and improving nutritional status and metabolic patterns play a key role in the application of conversion therapy on obstructive incurable AGC. 
Gastrojejunostomy is the standard surgical option to ameliorate GOO. Several studies have revealed that gastrojejunostomy can restore oral intake and enhance compliance with anticancer chemotherapies. ${ }^{7,8}$ In addition, laparoscopic gastrojejunostomy (LGJ) is a promising treatment option with multiple advantages, such as small incisions, reduced immunosuppression, and early gastrointestinal peristalsis recovery. ${ }^{7}$ However, nutritional status and metabolic pattern changes among patients who underwent LGJ followed by enteral nutrition have yet to be fully evaluated. Moreover, little is known regarding the feasibility and efficacy of LGJ when it is combined with conversion therapy in these patients.

Accordingly, the purpose of the present study was to clarify the feasibility and efficacy of multimodal therapy with LGJ followed by enteral nutrition and conversion therapy in patients with GOO caused by incurable AGC.

\section{Materials and Methods}

\section{Patient Selection}

We reviewed all cases of gastric outlet obstruction caused by incurable AGC treated in Fujian Provincial Hospital between January 2016 and September 2019. Eligibility criteria included histologic confirmation of gastric adenocarcinoma; presence of a non-curable factor; ${ }^{5}$ pyloric stenosis revealed by endoscopy and difficulty in oral intake due to stenosis; age between $20-80$ years; surgical fitness with adequate organ function; Eastern Cooperative Oncology Group (ECOG) performance status (PS) score 0-2; no prior chemotherapy, targeted therapy or radiotherapy. Patients with gastric cancer perforation or active bleeding requiring urgent surgery, pyloric stenosis caused by other diseases, other malignant tumours, or changes in chemotherapy regimens were excluded, as well as those who completed less than two chemotherapy cycles. This study was reviewed and approved by the Ethics Committee of Fujian Provincial Hospital. The data are anonymous, and the requirement for informed consent from patients was waived. All study procedures were performed in accordance with the Helsinki Declaration of 1964 and later versions.

\section{Treatment Regimes}

Patients with scores of $0 / 1$ on the gastric outlet obstruction scoring system (GOOSS) were categorized into multimodal therapy with LGJ before conversion therapy, while those with a GOOSS score of 2 were categorized in chemotherapy group. For patients receiving LGJ, operating trocars (5 or
$12 \mathrm{~mm}$ in diameter) were inserted for staging laparoscopy and cytological examination. Subsequently, the greater omentum, along the greater curvature, was dissected. Small incisions were created on the greater curvature, $5 \mathrm{~cm}$ proximal to the oral side of tumour and $15 \mathrm{~cm}$ distal to the ligament of Treitz on the antimesenteric side of the jejunum. The greater curvature was inosculated to the jejunum as sideto-side anastomosis, using a linear stapler.

After LGJ, enteral nutrition was initiated, combined with early parenteral nutrition. Patients were encouraged to drink a small amount of water on awakening after the surgery. On postoperative day 1 , patients started drinking $500-1000 \mathrm{~mL}$ clear fluid. The amount of fluid intake was increased gradually according to patients' tolerance. Parenteral nutrition was discontinued when oral intake reached 2000-2500 mL/d. During hospitalization, all patients (both groups) received nutrition (protein $4.0 \mathrm{~g}$, fat $3.9 \mathrm{~g}$, carbohydrate $12.1 \mathrm{~g}$, caloric value $1.0 \mathrm{kcal} / \mathrm{mL}$ ) at a temperature of $40^{\circ} \mathrm{C}$. The energy supply ranged between 25 and $30 \mathrm{kcal} / \mathrm{kg} /$ day, and protein intake was 1-2 $\mathrm{g} / \mathrm{kg} /$ day, supplemented by parenteral nutrition. For patients with severe malnutrition based on the PatientGenerated Subjective Global Assessment score (PG-SGA category C), ${ }^{9}$ the initial energy supply was limited to $5-10$ $\mathrm{kcal} / \mathrm{kg} /$ day, and energy intake increased slowly over 4-7 days until substitution of requirements was reached. ${ }^{10}$ Patients were allowed oral intake as tolerated after discharge.

Patients who underwent LGJ began palliative therapy 7-14 days after surgery. Palliative therapy in the multimodal therapy group and the chemotherapy group included EOX therapy, consisting of oral fluoropyrimidine capecitabine $(825 \mathrm{mg} / \mathrm{m} 2$, twice daily on days 1-14), intravenous oxaliplatin $(130 \mathrm{mg} / \mathrm{m} 2$ on day 1$)$, and intravenous epirubicin $(100 \mathrm{mg} / \mathrm{m} 2$ on day 1$)$. These treatments were generally continued for 6-8 cycles, until the occurrence of unacceptable toxicity, patients' refusal, or evidence of a resectable tumour. Patients received abdominal enhanced CT after every 2 cycles of chemotherapy. A multidisciplinary team assessed potentially resectable cases to determine the best timing of gastrectomy. After conversion surgery, patients started adjuvant chemotherapy according to the attending physician's judgment.

\section{Data Collection}

Data were retrospectively collected on patients' baseline characteristics, including the GOOSS, Nutritional Risk Screening 2002 (NRS-2002) $^{11}$ and PG-SGA assessments, nutritional and metabolic status, body composition, response to chemotherapy, and postoperative overall survival. Patients with a GOOSS score of $\leq 2$ were considered to have an 
eating disorder. ${ }^{7}$ Body composition ${ }^{12}$ was analysed by ImageJ2 software (The National Institutes of Health, Bethesda, MD, USA) to achieve the skeletal muscle area at diagnosis and after 2 chemotherapy cycles. The skeletal muscle index (SMI) was calculated by normalizing the skeletal muscle area for height $\left(\mathrm{cm}^{2} / \mathrm{m}^{2}\right)$. Sarcopenia was set at SMI lower than $43 \mathrm{~cm}^{2} / \mathrm{m}^{2}$ in patients with a body mass index $(\mathrm{BMI})<25 \mathrm{~kg} / \mathrm{m}^{2}$ and SMI lower than $53 \mathrm{~cm}^{2} / \mathrm{m}^{2}$ with BMI $>25 \mathrm{~kg} / \mathrm{m}^{2}$ in males; in females, sarcopenia was defined as $\mathrm{SMI}<41 \mathrm{~cm}^{2} / \mathrm{m}^{2}$, as reported in previous literature ${ }^{1,13}$ (Figure 1). Nutritional and metabolic status were estimated by Onodera's prognostic nutritional index (PNI) and the neutrophil to lymphocyte ratio (NLR). ${ }^{14,15}$ Quality of life (QOL) was evaluated with the Spitzer QOL-Index. ${ }^{16,17}$

Treatment responses were classified according to the response evaluation criteria in solid tumours guideline (version 1.0$)^{18}$ after every 2 cycles of chemotherapy. Toxicity was assessed according to the Common Toxicity Criteria for Adverse Events (version 3.0). ${ }^{19}$ The pathological response to chemotherapy was classified according to the National Comprehensive Cancer Network (NCCN) clinical practice guidelines in oncology for gastric cancer. $^{20}$ Complications were recorded until discharge after conversion surgery according to Clavien et al. ${ }^{2}$

\section{Statistical Analysis}

Continuous data were analysed using the Wilcoxon ranksum test. Categorical variables were analysed using the chi-square test or Fisher's exact test when appropriate When $\mathrm{P}$ values $<0.05$, the differences were considered significant. Overall survival (OS) was recorded as the duration from the date of initial chemotherapy to that of death or the latest follow-up. The OS was analysed by Kaplan-Meier method with Log rank test. Univariate and multivariate analyses for survival were performed using Cox's regression analysis. All statistical analysis was performed by SPSS software (IBM SPSS Statistics for Windows, version 22.0, Armonk, NY, USA).

\section{Results}

\section{Baseline Characteristics}

There were 101 patients treated for gastric outlet obstruction caused by incurable AGC during the study period. A total of 35 patients were excluded due to other treatments $(n=8)$, missing data $(n=8)$, changes in chemotherapy regimens $(\mathrm{n}=10)$, less than 2 chemotherapy cycles $(\mathrm{n}$ $=9$ ), leaving 66 patients for analysis. There were 35 patients who underwent multimodal therapy and 31 patients who underwent chemotherapy.

The baseline characteristics of all eligible patients are outlined in Table 1. The median age was 58 years (range, 28-80 years), and 43 were male. There were 34 patients in poor general condition (PS of 2). Further, 35, 65, 62, 48, and 46 patients had worse GOOSS (0 or 1), nutritional risk (NRS 2002 scale $>3$ ), severe malnutrition (PG-SGA category C), $\mathrm{PNI}<45$, and NLR $\geq 2.5$, respectively. The median overall

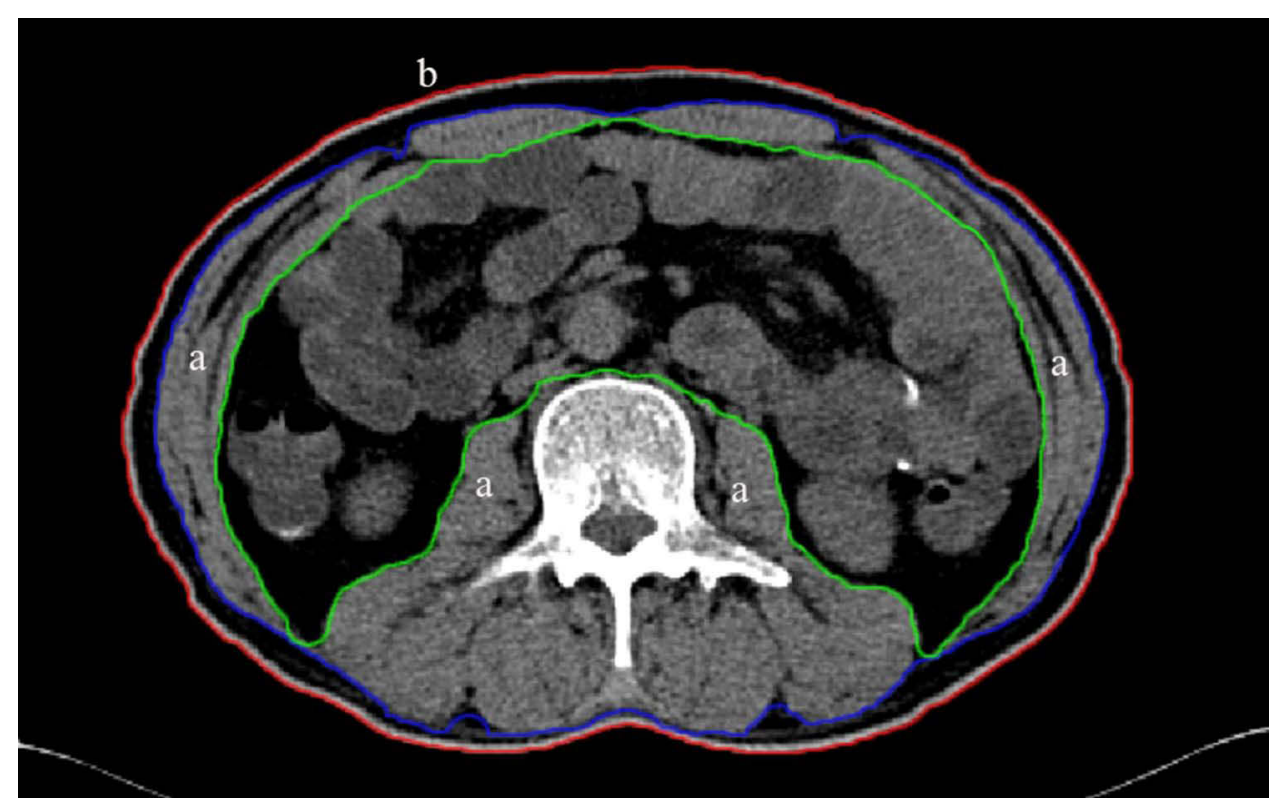

Figure I Example of transverse computed tomography images at 3rd lumbar vertebra. a: skeletal muscles area (between green and purple lines) b: abdominal perimeter (red line). 
Table I Baseline Patient Characteristics

\begin{tabular}{|c|c|}
\hline Characteristics & $N=66$ \\
\hline Age, (years)* & $58(28-80)$ \\
\hline Sex, (male/female) & $43 / 23(65.2 \% / 34.8 \%)$ \\
\hline Performance status, (0/I/2) & $4 / 28 / 34(6.1 \% / 42.4 \% / 51.5 \%)$ \\
\hline GoOSS, (0/I/2) & $|5 / 20 / 3|(22.7 \% / 30.3 \% / 47.0 \%)$ \\
\hline \multicolumn{2}{|l|}{ NRS 2002 scale } \\
\hline$\leq 3$ & I (I.5\%) \\
\hline$>3$ & 65 (98.5\%) \\
\hline \multicolumn{2}{|l|}{ PG-SGA category } \\
\hline B & $4(6.1 \%)$ \\
\hline $\mathrm{C}$ & $62(93.9 \%)$ \\
\hline \multicolumn{2}{|l|}{ PNI } \\
\hline$<45$ & $48(72.7 \%)$ \\
\hline$\geq 45$ & $18(27.3 \%)$ \\
\hline \multicolumn{2}{|l|}{ NLR } \\
\hline$<2.5$ & $20(30.3 \%)$ \\
\hline$\geq 2.5$ & $46(69.7 \%)$ \\
\hline Overall QOL* & $6(4-9)$ \\
\hline \multicolumn{2}{|l|}{ Body composition } \\
\hline BMI $\left(\mathrm{kg} / \mathrm{m}^{2}\right)^{*}$ & $21.7(17.3-26.4)$ \\
\hline SMI $\left(\mathrm{cm}^{2} / \mathrm{m}^{2}\right)^{*}$ & $48.8(33.4-65.4)$ \\
\hline Sarcopenia & $24(36.4 \%)$ \\
\hline \multicolumn{2}{|l|}{ cT } \\
\hline T3 & $6(9.1 \%)$ \\
\hline $\mathrm{T} 4$ & $60(90.9 \%)$ \\
\hline $\mathrm{cN}(+)$ & $66(100 \%)$ \\
\hline \multicolumn{2}{|l|}{ Non-curable factor } \\
\hline $\begin{array}{l}\text { Tumour infiltration to adjacent } \\
\text { organs }\end{array}$ & $7(10.6 \%)$ \\
\hline Peritoneal metastasis & $35(53.0 \%)$ \\
\hline Hepatic metastasis & $16(24.2 \%)$ \\
\hline Distant lymph nodes metastasis & $39(59.1 \%)$ \\
\hline \multicolumn{2}{|l|}{ Laparoscopic gastrojejunostomy } \\
\hline Present & $35(53.0 \%)$ \\
\hline Absent & $31(47.0 \%)$ \\
\hline
\end{tabular}

Note: *Median (range).

Abbreviations: GOOSS, gastric outlet obstruction scoring system; 0, no oral intake; I, liquids only; 2, soft food, 3, solid food; NRS 2002, Nutrition Risk Screening 2002; PG-SGA, Patient-Generated Subjective Global Assessment; PNI, Prognostic Nutritional Index; NLR, Neutrophil to lymphocyte ratio; QOL, Quality of life; BMI, body mass index; SMI, skeletal muscle index.

QOL of all patients was 6. Thirty one (47.0\%) patients had $>1$ non-curable factors. The primary reasons for unresectability included infiltration to adjacent organs $(\mathrm{n}=7,10.6 \%)$, peritoneal metastasis $(\mathrm{n}=35,53.0 \%$, including 3 patients with positive peritoneal lavage cytology determined by staging laparoscopy), hepatic metastasis $(\mathrm{n}=16,24.2 \%)$, and distant lymph node $(\mathrm{LN})$ metastasis $(\mathrm{n}=39,59.1 \%$, including 32 para-aortic, 6 mediastinal, and 1 Virchow's LN).

The baseline demographic and clinical characteristics of the multimodal therapy and chemotherapy groups are compared in Table 2. No significant differences were detected in age, sex, PS, NRS 2002 scale, PG-SGA category, overall QOL score, body composition, clinical stage, or non-curable factors. Baseline GOOSS was better in the multimodal therapy group in comparison to the chemotherapy group $(P<0.001)$.

\section{Patient Treatment Outcomes}

Clinical outcomes were collected after 2 cycles of chemotherapy (Table 3). Most patients resumed solid food intake 7 days after LGJ. In the multimodal therapy group, $34(97.1 \%)$ patients had a GOOSS score of 3 after chemotherapy. Of note, the rates of nutritional risk, severe malnutrition, and PNI $<45$ were significantly lower in the multimodal therapy group than in the chemotherapy group ( $17.1 \%$ vs $96.8 \%$, 0 vs $96.8 \%$, and $28.6 \%$ vs $80.6 \%$, respectively, $P<0.001)$. Further, the overall QOL was also significantly higher in multimodal therapy patients (16.7 months vs 4.5 months, $P<0.001)$. However, no significant difference was observed in the NLR scores between the groups $(P=0.454)$. More than half $(54.8 \%)$ of the patients in the chemotherapy group had sarcopenia after treatment, in contrast to only $25.6 \%$ of the patients in the multimodal therapy group $(P=0.030)$.

Among those who received multimodal therapy, 17 patients $(48.6 \%)$ displayed a major response (3 complete responses and 14 partial responses), which was significantly better than the response rate in the chemotherapy group $(P<0.001)$. Multimodal therapy patients received more cycles of chemotherapy (6 cycles vs 3 cycles, $P<$ $0.001)$. The non-curable factors disappeared after chemotherapy for 17 patients $(48.6 \%)$ in the multimodal therapy group, and these patients all underwent subsequent gastrectomy. None of the chemotherapy group patients had resolution of non-curable factors. No significant differences were observed between the groups in chemotherapy-related toxicities.

\section{Surgery and Pathologic Results}

Conversion surgery was performed in 17 (48.6\%) multimodal therapy patients, whereas none of chemotherapy patients received the conversion surgery. All patients 
Table 2 Comparison of Baseline Characteristics for Multimodal Therapy and Chemotherapy Groups

\begin{tabular}{|c|c|c|c|}
\hline Characteristics & Multimodal Therapy $(n=35)$ & Chemotherapy $(n=31)$ & $P$ value \\
\hline Age, (years)* & $63(35-80)$ & $57(28-74)$ & 0.131 \\
\hline Sex, (male/female) & $25 / 10(71.4 \% / 28.6 \%)$ & $|8 /| 3(58.1 \% / 4 \mid .9 \%)$ & 0.255 \\
\hline Performance status, (0/I/2) & $2 / 17 / 16(5.7 \% / 48.6 \% / 45.7 \%)$ & $2 / I I / / 8(6.5 \% / 35.5 \% / 58.0 \%)$ & 0.377 \\
\hline GoOSS, $(0 / 1 / 2)$ & $15 / 20 / 0(42.9 \% / 57.1 \% / 0)$ & $0 / 0 / 31(0 / 0 / 100 \%)$ & $<0.001$ \\
\hline NRS 2002 scale & & & 0.530 \\
\hline$\leq 3$ & $\mathrm{I}(2.9 \%)$ & $0(0)$ & \\
\hline$>3$ & $34(97.1 \%)$ & $31(100 \%)$ & \\
\hline PG-SGA category & & & 0.603 \\
\hline B & $3(8.6 \%)$ & $3(9.7 \%)$ & \\
\hline C & $32(91.4 \%)$ & $28(90.3 \%)$ & \\
\hline PNI & & & 0.801 \\
\hline$<45$ & $25(71.4 \%)$ & $23(74.2 \%)$ & \\
\hline$\geq 45$ & $10(28.6 \%)$ & $8(25.8 \%)$ & \\
\hline NLR & & & 0.389 \\
\hline$<2.5$ & $9(25.7 \%)$ & II (35.5\%) & \\
\hline$\geq 2.5$ & $26(74.3 \%)$ & $20(64.5 \%)$ & \\
\hline Overall QOL* & $6(4-9)$ & $6(4-8)$ & 0.123 \\
\hline \multicolumn{4}{|l|}{ Body composition } \\
\hline BMI $\left(\mathrm{kg} / \mathrm{m}^{2}\right)^{*}$ & $21.2(17.3-26.4)$ & $22.3(18.2-24.3)$ & 0.230 \\
\hline SMI $\left(\mathrm{cm}^{2} / \mathrm{m}^{2}\right)^{*}$ & $49.5(36.5-65.4)$ & $48.6(30.0-65.4)$ & 0.979 \\
\hline Sarcopenia & $14(40.0 \%)$ & $10(32.3 \%)$ & 0.514 \\
\hline cT & & & 0.442 \\
\hline T3 & $2(5.7 \%)$ & $3(9.7 \%)$ & \\
\hline $\mathrm{T} 4$ & $33(94.3 \%)$ & $28(90.3 \%)$ & \\
\hline $\mathrm{cN}(+)$ & $35(100 \%)$ & $31(100 \%)$ & I \\
\hline \multicolumn{4}{|l|}{ Non-curable factor } \\
\hline Infiltration to adjacent organs & $3(8.6 \%)$ & $4(12.9 \%)$ & 0.431 \\
\hline Peritoneal metastasis & $20(57.1 \%)$ & $15(48.4 \%)$ & 0.477 \\
\hline Hepatic metastasis & $8(22.9 \%)$ & $8(25.8 \%)$ & 0.780 \\
\hline Distant lymph nodes metastasis & $19(54.3 \%)$ & $20(64.5 \%)$ & 0.399 \\
\hline
\end{tabular}

Note: *Median (range).

Abbreviations: GOOSS, gastric outlet obstruction scoring system; NRS 2002, Nutrition Risk Screening 2002; PG-SGA, Patient-Generated Subjective Global Assessment; PNI, Prognostic Nutritional Index; NLR, Neutrophil to lymphocyte ratio; QOL, Quality of life; BMI, body mass index; SMI, skeletal muscle index.

underwent distal gastrectomy and D2 LN dissection, followed by postoperative chemotherapy. R0 resection was achieved in $15(88.2 \%)$ while $\mathrm{R} 1 / \mathrm{R} 2$ resection in 2 $(11.8 \%)$ (Table 4) patients. Among the 7 patients with peritoneal metastasis, staging laparoscopy was performed, confirming the disappearance of metastasis. Additional radiofrequency ablation was applied to hepatic metastasis of 4 patients, resulting in complete disappearance of metastatic lesions. In the 3 patients with infiltration to adjacent organs, the metastatic lesions disappeared after chemotherapy. The pathologic responses were tumour regression grade (TRG) $0,1,2$, and 3 in 3, 4, 6, and 4 patients, respectively. Operation-related complications were observed in $2(11.8 \%)$ patients, which were all cured by conservative treatments.

\section{Survival Analyses}

The median survival time (MST) was 7.5 months in terms of all patients (Figure 2). The MST in the multimodal therapy group was 16.7 months, compared to 4.5 months 
Table 3 Treatment Outcomes for Multimodal Therapy and Chemotherapy Groups

\begin{tabular}{|c|c|c|c|}
\hline Characteristics & Multimodal Therapy $(n=35)$ & Chemotherapy $(n=31)$ & $P$ value \\
\hline GOOSS 3 achieved & $34(97.1 \%)$ & $0(0)$ & $<0.001$ \\
\hline Chemotherapy cycles* & $6(2-10)$ & $3(2-6)$ & 0.005 \\
\hline NRS 2002 scale & & & $<0.001$ \\
\hline$\leq 3$ & $29(82.9 \%)$ & $\mathrm{I}(3.2 \%)$ & \\
\hline$>3$ & $6(17.1 \%)$ & $30(96.8 \%)$ & \\
\hline PG-SGA category & & & $<0.001$ \\
\hline$A$ & $15(42.9 \%)$ & $0(0)$ & \\
\hline B & $20(57.1 \%)$ & $\mathrm{I}(3.2 \%)$ & \\
\hline C & $0(0)$ & $30(96.8 \%)$ & \\
\hline PNI & & & $<0.001$ \\
\hline$<45$ & $10(28.6 \%)$ & $25(80.6 \%)$ & \\
\hline$\geq 45$ & $25(71.4 \%)$ & $6(19.4 \%)$ & \\
\hline NLR & & & 0.454 \\
\hline$<2.5$ & $23(65.7 \%)$ & $23(74.2 \%)$ & \\
\hline$\geq 2.5$ & $12(34.3 \%)$ & $8(25.8 \%)$ & \\
\hline Overall QOL* & $9(7-10)$ & $6(4-9)$ & $<0.001$ \\
\hline \multicolumn{4}{|l|}{ Body composition } \\
\hline BMI $\left(\mathrm{kg} / \mathrm{m}^{2}\right)^{*}$ & $20.8(17.4-27.0)$ & $21.6(17.9-25.4)$ & 0.183 \\
\hline SMI $\left(\mathrm{cm}^{2} / \mathrm{m}^{2}\right)^{*}$ & $49.1(39.3-66.2)$ & $42.3(30.0-69.2)$ & 0.036 \\
\hline Sarcopenia & $10(25.6 \%)$ & $17(54.8 \%)$ & 0.030 \\
\hline \multicolumn{4}{|l|}{ Response } \\
\hline Complete response & $3(8.6 \%)$ & $0(0)$ & \\
\hline Partial response & |4(40.0\%) & $2(6.5 \%)$ & \\
\hline Stable disease & $8(22.8 \%)$ & $17(54.8 \%)$ & \\
\hline Progressive disease & $10(28.6 \%)$ & $12(38.7 \%)$ & \\
\hline ORR & $48.6 \%$ & $6.5 \%$ & $<0.001$ \\
\hline \multicolumn{4}{|l|}{ Adverse events (grade $3 / 4$ ) } \\
\hline Anemia & $6(17.1 \%)$ & $4(12.9 \%)$ & 0.448 \\
\hline Neutropenia & $18(51.4 \%)$ & $15(48.4 \%)$ & 0.805 \\
\hline Thrombocytopenia & $4(11.4 \%)$ & $4(12.9 \%)$ & 0.574 \\
\hline Elevated ALT & $2(5.7 \%)$ & $0(0)$ & 0.277 \\
\hline Elevated AST & $2(5.7 \%)$ & $0(0)$ & 0.277 \\
\hline Diarrhea & $\mathrm{I}(2.9 \%)$ & $2(6.5 \%)$ & 0.454 \\
\hline Subsequent resection & $17(48.6 \%)$ & $0(0)$ & $<0.001$ \\
\hline
\end{tabular}

Note: *Median (range).

Abbreviations: GOOSS, gastric outlet obstruction scoring system; NRS 2002, Nutrition Risk Screening 2002; PG-SGA, Patient-Generated Subjective Global Assessment; PNI, Prognostic Nutritional Index; NLR, Neutrophil to lymphocyte ratio; QOL, Quality of life; BMI, body mass index; SMI, skeletal muscle index; ORR, Objective Response Rate.

for the chemotherapy group. Further, the 1- and 3-year OS rates in the multimodal therapy group were $68.6 \%$ and $37.1 \%$, respectively, compared to $6.5 \%$ and $0 \%$ in the chemotherapy group. Furthermore, the MST of patients receiving conversion surgery was significantly better than that of patients without surgery (44.2 months vs 8.5 months, respectively, $P<0.001)$. The 15 patients $(88.2 \%)$ who achieved R0 resection had a higher 3-year
OS rate of $86.7 \%$ (MST 45.8 months), while the 2 patients who achieved R1/R2 resection had a 3-year OS rate of $0 \%$. During follow-up, recurrence in the liver was observed in 2 patients treated by conversion therapy, the OS was 14.3 months and 18.3 months respectively. Complete remission was achieved in 3 patients (peritoneal metastasis only, $\mathrm{n}=1$; distant $\mathrm{LN}$ metastasis only, $\mathrm{n}=1$; peritoneal and $\mathrm{LN}$ metastasis, $\mathrm{n}=1$ ), all of whom 
Table 4 Surgical and Pathological Findings for Conversion Surgery Patients

\begin{tabular}{|c|l|l|}
\hline & $\mathbf{n}$ & $\%$ \\
\hline $\begin{array}{c}\text { Resection margin } \\
\text { R0 }\end{array}$ & 15 & 88.2 \\
RI/R2 & 2 & 11.8 \\
\hline Pathological response & & \\
0 & 3 & 17.7 \\
I & 4 & 23.5 \\
2 & 6 & 35.3 \\
3 & 4 & 23.5 \\
\hline PT & & \\
T0 & 3 & 17.7 \\
T3 & 2 & 11.8 \\
T4a & 12 & 70.5 \\
\hline PN & & \\
N0 & 14 & 82.4 \\
NI & 3 & 17.6 \\
\hline PM & 17 & \\
M0 & 17 & 5.9 \\
\hline Complications & 100 \\
Wound infection & & \\
Leakage & 17 & \\
\hline Postoperative chemotherapy & & \\
Present & & \\
\hline
\end{tabular}

subsequently underwent surgery, achieving a 3-year OS rate of $100 \%$.

Univariate analysis of all patients identified multimodal therapy as a significant prognostic factor. Other variables, such as BMI, PS, and overall QOL were not significant prognostic factors. Multivariate analysis identified multimodal therapy and improved or stable overall QOL as independent factors for OS (Table 5).

\section{Discussion}

Clinically relevant prognostic factors for LGJ combined with conversion therapy in patients with GOO caused by incurable AGC have not been well elucidated. To the best of our knowledge, this study is the first to investigate these factors and assess the feasibility and efficacy of this multimodal therapy. The results of this study demonstrated a marked improvement in eating function after LGJ, similar to a previous study, ${ }^{7}$ suggesting that LGJ is an effective therapeutic method to restore oral intake. The nutritional status, QOL, and response to chemotherapy in the multimodal therapy group was markedly better than that in the chemotherapy group. Further, the multimodal therapy group had less muscle loss after treatment than the chemotherapy group, leading to more cycles of chemotherapy. The MST of multimodal therapy group was also higher than that of chemotherapy group, especially in patients receiving conversion surgery. Multivariate analysis identified multimodal therapy and improved or stable overall QOL as independent factors for OS. This phenomenon may be related to better nutritional and metabolic status after restoration of enteral nutrition. These findings suggest that this multimodal therapy is potentially beneficial for the long-term prognosis in such patients.

Previous studies on treatment therapy for malignant GOO caused by AGC have assessed palliative gastrectomy, stent, and gastrojejunostomy. The REGATTA trial revealed that palliative gastrectomy had no survival benefit over chemotherapy alone, ${ }^{21}$ which may have been due to impaired compliance with chemotherapy after gastrectomy. Furthermore, Jonathan et $\mathrm{al}^{22}$ reported that cancer cells might disseminate via haematogenous and lymphatic routes after palliative surgery. Similar findings were reported in many investigations, ${ }^{23,24}$ suggesting poor prognosis with palliative surgery. However, Sunguk et $\mathrm{al}^{8}$ observed that gastrojejunostomy could result in longer survival. With the introduction of laparoscopic technique, LGJ would be the first choice to improve survival in GOO patients. ${ }^{25}$ Interestingly, in this study, no significant differences were observed in baseline inflammatory and nutritional status, whereas the multimodal therapy group had lower GOOSS. This phenomenon may be related to early detection owing to severe gastric outlet obstruction.

Furthermore, many investigators ${ }^{2-4,18}$ demonstrated that long-term survivors were observed in patients who received conversion surgery. Kazuya et $\mathrm{al}^{3}$ reported that the median survival time was extended to 41.3 months in R0 resected patients. Most conversion trials have targeted patients without GOO, and conversion therapy may not achieve such positive outcomes in these patients. A study by Tsuyoshi et $\mathrm{al}^{7}$ included only 4 patients $(13.3 \%)$ who underwent radical gastrectomy after conversion chemotherapy in GOO caused by incurable AGC. This may be due to malnutrition and metabolic disorder caused by a lengthy period without oral feeding in GOO patients. Therefore, the present study used various indicators to explore changes in nutritional and metabolic status after restoring oral intake, verifying the reliability and potentiality of this multimodal therapy. 


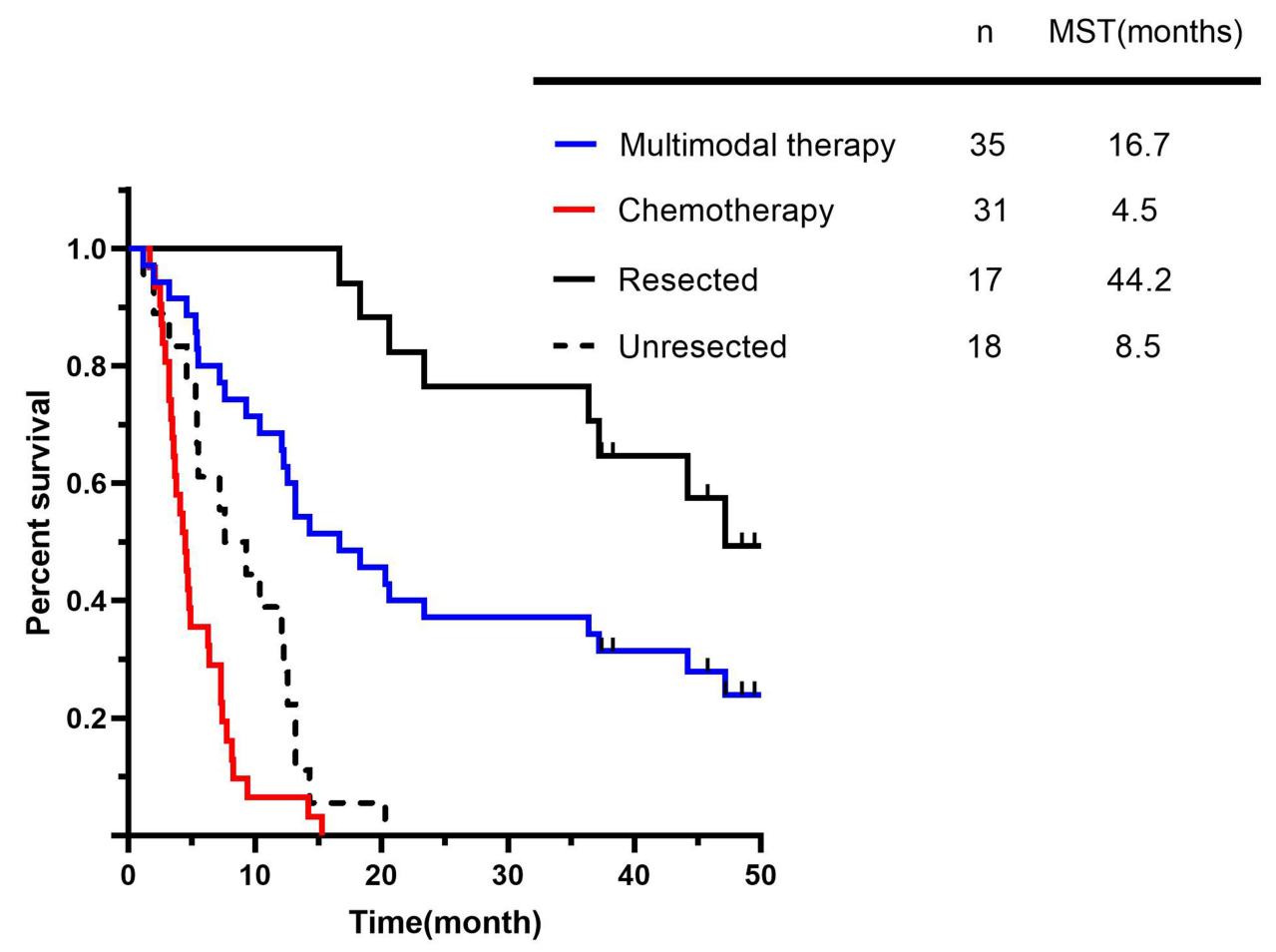

Figure 2 Survival curve of all patients enrolled in the current analysis. The MST of the patients with LGJ combined with conversion therapy (Multimodal therapy) was 16.7 months, and it was 4.5 months in those with chemotherapy only (Chemotherapy). The MST of resected patients (Resected) was 44.2 months, and the MST of unresected patients in multimodal therapy (Unresected) was 8.5 months.

The present study revealed that patients had a significantly lower rate of nutritional risk, severe malnutrition, and PNI $<45$ after multimodal therapy as compared to chemotherapy alone. The NRS2002, PG-SGA, and PNI are the most frequently used scales in nutritional risk screening and assessment for gastric cancers. These comprehensive screening methods are well-correlated with treatment and survival. ${ }^{11,26-28}$ Previous research has

Table 5 Univariate and Multivariate Analyses for Overall Survival

\begin{tabular}{|l|l|l|l|}
\hline Variable & Hazard Ratio & $\mathbf{9 5 \%} \mathbf{~ I ~}$ & $\boldsymbol{P}$ value \\
\hline Univariate analysis & & & \\
Multimodal therapy (present/absent) & 5.966 & $3.138-11.342$ & $<0.00$ I \\
Age $(\geq 65 /<65)^{*}$ & 1.321 & $0.765-2.280$ & 0.318 \\
BMI $(\geq 18.5 /<18.5)^{*}$ & 1.039 & $0.413-2.612$ & 0.935 \\
Performance status (2/0 or I)* & 0.802 & $0.475-1.354$ & 0.410 \\
PG-SGA category (B/C)* & 1.055 & $0.378-2.946$ & 0.918 \\
PNI ( $\geq 45 /<45)^{*}$ & 0.922 & $0.517-1.642$ & 0.782 \\
NLR ( $\geq 2.5 /<2.5)^{*}$ & 1.331 & $0.762-2.325$ & 0.314 \\
Overall QOL (improved or stable/decreased) & 0.679 & $0.291-1.587$ & 0.371 \\
Sarcopenia (yes/no)* & 0.872 & $0.508-1.497$ & 0.619 \\
\hline Multivariate analysis & & & $<0.001$ \\
Multimodal therapy (present/absent) & 8.199 & $4.076-16.492$ & 0.742 \\
Age( $\geq 65 /<65)^{*}$ & 1.098 & $0.631-1.910$ & 0.024 \\
Overall QOL (improved or stable/decreased) & 0.366 & $0.152-0.878$ & 0.243 \\
Sarcopenia (yes/no)* & 0.717 & $0.410-1.254$ & \\
\hline
\end{tabular}

Note: *Data at baseline.

Abbreviations: BMI, body mass index; GOOSS, gastric outlet obstruction scoring system; PG-SGA, Patient-Generated Subjective Global Assessment; PNI, Prognostic Nutritional Index; NLR, Neutrophil to lymphocyte ratio; QOL, Quality of life. 
reported that $40-80 \%$ of patients with malignant tumours experience malnutrition because of tumour growth and inappetence. $^{27}$ GOO and subsequent treatment undoubtedly exacerbate malnutrition, accelerating disease progression. Sa-Hong et $\mathrm{al}^{25}$ revealed that patients could tolerate chemotherapy longer after LGJ. This may be due to resumption of oral intake. AGC is characterized by chronic malnutrition, impairing the quality of life and survival. Resumption of oral intake could not only ameliorate malnutrition but also repair the gastrointestinal barrier, prevent migration of bacteria, and restore atrophic mucosa. $^{29}$

Another major concern in AGC is systemic inflammation. Tumours have been considered as "wounds that do not heal", as a systemic inflammatory response would be initiated by stromal inflammatory mediators in the systemic circulation. $^{28}$ A previous study ${ }^{29}$ indicated that enteral nutrition could alleviate inflammatory response through regulating gastrointestinal immune system. However, our findings revealed no difference in the two treatment groups in terms of NLR. The reason may be that cytotoxic anticancer chemotherapy has myelosuppressive effects, especially neutropenia, ${ }^{30}$ at the initiation of therapy.

Numerous analyses have reported that patients with excellent nutritional status could be more treatment compliant, with higher rates of chemotherapy and operation. ${ }^{12,31-34}$ A propensity score-matched analysis of gastrojejunostomy revealed that most patients were able to consume normal diet intake (95\% achieved a GOOSS score of 3 ), and found improved oral intake to be a significant prognostic factor. ${ }^{6}$ Moreover, a study of LGJ combined with conversion chemotherapy for patients with GOO reported that the MST for these patients was superior than that in the best supportive care group (35.9 months vs 12.2 months). ${ }^{7}$ Therefore, LGJ followed by enteral nutrition might be a promising treatment for conversion therapy for incurable obstructive gastric cancers.

In this study, non-curative factors disappeared in 17 patients $(48.6 \%)$ in the multimodal therapy group, and these patients were subsequently able to receive conversion surgery. In comparison, no patients received conversion surgery in the chemotherapy group. In addition, the objective response rate was high in the multimodal therapy group, similar to previous research results. ${ }^{18}$ The proportion of resected patients in the multimodal therapy group was $48.6 \%$, with a high pathological response rate of $76.5 \%$ (TRG 0-2). These response rates may have resulted in a good prognosis, and the MST of resected cases was
44.2 months, which was significantly higher than that of both the chemotherapy cases (4.5 months) and the unresected cases in the multimodal therapy group ( 8.5 months). This observation highlights the vital role that the multimodal therapy could play in the future, as these patients were originally best considered for supportive care only, without any hope of long-term survival.

Conversion therapy necessitates careful observation of chemotherapy toxicity, especially in patients with sarcopenia. Cancer cachexia, diminished food intake, and activation of catabolic pathways will contribute to muscle loss. ${ }^{35}$ Sherif et $\mathrm{al}^{33}$ revealed that over half of patients with esophagogastric cancer had sarcopenia prior to chemotherapy, and even more patients were affected on completion of chemotherapy. In our study, we observed that the proportion of patients with sarcopenia was significantly lower in the multimodal therapy group than in the chemotherapy group. It is a reminder that adequate nutritional support may prevent or even reverse muscle loss. Aoife et $\mathrm{al}^{35}$ and Tan et $\mathrm{al}^{34}$ had demonstrated that sarcopenia increases the risk of toxicity. Our study revealed that multimodal treatment therapy was associated with fewer adverse events, and especially less neutropenia, than inoperable gastric cancers with conversion therapy alone in a previous study. ${ }^{18}$ However, no significant differences were observed between our 2 treatment groups, probably due to the lower number of chemotherapy cycles in the chemotherapy group. The postoperative complications rate $(9.6 \%)$ in our study was lower than that in gastric cancer patients receiving conversional radical surgery in a previous research, ${ }^{36}$ which may result from better nutritional status. In summary, these findings demonstrate that this multimodal therapy is safe and feasible.

Several limitations of the present study are worth noting. First, the study analysed a relatively small sample size. Moreover, it was limited by its retrospective exploratory design. Previous studies also had these limitations, due to the rarity of GOO in gastric cancer. However, this study included a comprehensive analysis of multiple disease indicators. Second, we did not assess the nutritional status and survival of patients with less than 2 chemotherapy cycles or without treatment. Since the target patients had obstructive incurable AGC, many patients declined further treatment. Difficulties were also associated with obtaining sequential data in these patients. Finally, the follow-up period in this study was not long enough to determine the 5-year survival rate. 


\section{Conclusion}

This study analysed multiple clinical outcomes to demonstrate that multimodal therapy with LGJ followed by enteral nutrition and conversion therapy was feasible and effective in treating GOO caused by incurable AGC. A large-scale randomized prospective study is needed to confirm the therapeutic usefulness and extend the application scope of this multimodal therapy.

\section{Data Sharing Statement}

Please contact the corresponding author (Weihua Li, email: liwh@fjmu.edu.cn) for data requests.

\section{Ethics Approval and Consent to Participate}

This study was approved by the Ethics Committee of Fujian Provincial Hospital.

\section{Acknowledgments}

We wish to thank all colleagues and nurses who provided care to the patients in this study.

\section{Funding}

No funding was received.

\section{Disclosure}

All authors report no conflicts of interest in this work.

\section{References}

1. Dijksterhuis WPM, Pruijt MJ, van der Woude SO, et al. Association between body composition, survival, and toxicity in advanced esophagogastric cancer patients receiving palliative chemotherapy. J Cachexia Sarcopenia Muscle. 2019;10(1):199-206. doi:10.1002/ jcsm. 12371

2. Solaini L, Ministrini S, Bencivenga M, et al. Conversion gastrectomy for stage IV unresectable gastric cancer: a GIRCG retrospective cohort study. Gastric Cancer. 2019;22(6):1285-1293. doi:10.1007/s10120019-00968-2

3. Ministrini S, Bencivenga M, Solaini L, et al. Stage IV gastric cancer: the surgical perspective of the Italian research group on gastric cancer. Cancers. 2020;12(1). doi:10.3390/cancers12010158.

4. Yoshida K, Yamaguchi K, Okumura N, Tanahashi T, Kodera Y. Is conversion therapy possible in stage IV gastric cancer: the proposal of new biological categories of classification. Gastric Cancer. 2016;19 (2):329-338. doi:10.1007/s10120-015-0575-Z

5. Zhang F, Huang X, Song Y, et al. Conversion Surgery for Stage IV Gastric Cancer. Front Oncol. 2019;9:1158. doi:10.3389/ fonc. 2019.01158

6. Haga Y, Hiki N, Kinoshita T, et al. Treatment option of endoscopic stent insertion or gastrojejunostomy for gastric outlet obstruction due to gastric cancer: a propensity score-matched analysis. Gastric Cancer. 2020;23(4):667-676. doi:10.1007/s10120-020-01040-0
7. Tanaka T, Suda K, Satoh S, et al. Effectiveness of laparoscopic stomach-partitioning gastrojejunostomy for patients with gastric outlet obstruction caused by advanced gastric cancer. Surg Endosc. 2017;31(1):359-367. doi:10.1007/s00464-016-4980-0

8. Jang S, Stevens T, Lopez R, Bhatt A, Vargo JJ. Superiority of Gastrojejunostomy Over Endoscopic Stenting for Palliation of Malignant Gastric Outlet Obstruction. Clin Gastroenterol Hepatol. 2019;17(7):1295-1302.e1291. doi:10.1016/j.cgh.2018.10.042

9. Dong J, Zhang W, Zhang T, et al. Baseline nutritional status could be a predictor for radiation esophagitis in esophageal cancer patients undergoing radiotherapy. Ann Transl Med. 2020;8(18):1148. doi: $10.21037 / \mathrm{atm}-20-4078$

10. Arends J, Bachmann P, Baracos V, et al. ESPEN guidelines on nutrition in cancer patients. Clin nutr. 2017;36(1):11-48.

11. Li YF, Nie RC, Wu T, et al. Prognostic Value of the Nutritional Risk Screening 2002 Scale in Metastatic Gastric Cancer: a Large-Scale Cohort Study. J Cancer. 2019;10(1):112-119. doi:10.7150/jca.27729

12. Palmela C, Velho S, Agostinho L, et al. Body Composition as a Prognostic Factor of Neoadjuvant Chemotherapy Toxicity and Outcome in Patients with Locally Advanced Gastric Cancer. J Gastric Cancer. 2017;17(1):74-87. doi:10.5230/jgc.2017.17.e8

13. Gomez-Perez SL, Haus JM, Sheean P, et al. Measuring Abdominal Circumference and Skeletal Muscle From a Single Cross-Sectional Computed Tomography Image: a Step-by-Step Guide for Clinicians Using National Institutes of Health ImageJ. JPEN J Parenter Enteral Nutr. 2016;40(3):308-318. doi:10.1177/0148607115604149

14. Mimatsu K, Fukino N, Ogasawara Y, Saino Y, Oida T. Utility of Inflammatory Marker- and Nutritional Status-based Prognostic Factors for Predicting the Prognosis of Stage IV Gastric Cancer Patients Undergoing Non-curative Surgery. Anticancer Res. 2017;37 (8):4215-4222.

15. Yoshida N, Baba Y, Shigaki H, et al. Preoperative Nutritional Assessment by Controlling Nutritional Status (CONUT) is Useful to estimate Postoperative Morbidity After Esophagectomy for Esophageal Cancer. World J Surg. 2016;40(8):1910-1917. doi:10.1007/s00268-016-3549-3

16. Terashima M, Fujitani K, Ando M, et al. Survival analysis of a prospective multicenter observational study on surgical palliation among patients receiving treatment for malignant gastric outlet obstruction caused by incurable advanced gastric cancer. Gastric Cancer. 2021;24(1):224-231. doi:10.1007/s10120-020-01114-z

17. Pulenzas N, Khan L, Tsao M, et al. Fatigue scores in patients with brain metastases receiving whole brain radiotherapy. Supportive Care Cancer. 2014;22(7):1757-1763. doi:10.1007/s00520-014-2140-4

18. Sato Y, Ohnuma H, Nobuoka T, et al. Conversion therapy for inoperable advanced gastric cancer patients by docetaxel, cisplatin, and S-1 (DCS) chemotherapy: a multi-institutional retrospective study. Gastric Cancer. 2017;20(3):517-526. doi:10.1007/s10120-016-0633-1

19. Zhou C, Ma T, Shi M, et al. Dose-finding study of modified FLOT (mFLOT) regimen as first-line treatment in Chinese patients with metastatic adenocarcinoma of stomach. Cancer Chemother Pharmacol. 2020;85(1):113-119. doi:10.1007/s00280-019-03982-4

20. Provenzale D, Gupta S, Ahnen DJ, et al. Genetic/Familial High-Risk Assessment: colorectal Version 1.2016, NCCN Clinical Practice Guidelines in Oncology. J Nat Comprehensive Cancer Network. 2016;14(8):1010-1030. doi:10.6004/jnccn.2016.0108

21. Fujitani K, Yang HK, Mizusawa J, et al. Gastrectomy plus chemotherapy versus chemotherapy alone for advanced gastric cancer with a single non-curable factor (REGATTA): a Phase 3, randomised controlled trial. Lancet Oncol. 2016;17(3):309-318. doi:10.1016/ S1470-2045(15)00553-7

22. Hiller JG, Perry NJ, Poulogiannis G, Riedel B, Sloan EK. Perioperative events influence cancer recurrence risk after surgery. Nat Rev Clin Oncol. 2018;15(4):205-218. doi:10.1038/ nrclinonc.2017.194 
23. Krall JA, Reinhardt F, Mercury OA, et al. The systemic response to surgery triggers the outgrowth of distant immune-controlled tumours in mouse models of dormancy. Sci Transl Med. 2018;10:436. doi:10.1126/scitranslmed.aan3464

24. Lange PH, Hekmat K, Bosl G, Kennedy BJ, Fraley EE. Acclerated growth of testicular cancer after cytoreductive surgery. Cancer. 1980;45(6):1498-1506. doi:10.1002/1097-0142(19800315) 45:6<1498::AID-CNCR2820450633>3.0.CO;2-7

25. Min SH, Son SY, Jung DH, et al. Laparoscopic gastrojejunostomy versus duodenal stenting in unresectable gastric cancer with gastric outlet obstruction. Ann Surg Treatment Res. 2017;93(3):130-136. doi:10.4174/astr.2017.93.3.130

26. Meng Q, Tan S, Jiang Y, et al. Post-discharge oral nutritional supplements with dietary advice in patients at nutritional risk after surgery for gastric cancer: a randomized clinical trial. Clin nutr. 2021;40 (1):40-46.

27. Seo SH, Kim S-E, Kang Y-K, et al. Association of nutritional status-related indices and chemotherapy-induced adverse events in gastric cancer patients. BMC Cancer. 2016;16(1):900. doi:10.1186/ s12885-016-2934-5

28. Arends J. Struggling with nutrition in patients with advanced cancer: nutrition and nourishment-focusing on metabolism and supportive care. Ann Oncol. 2018;29(suppl_2):ii27-ii34. doi:10.1093/annonc/ mdy093

29. Schörghuber M, Fruhwald S. Effects of enteral nutrition on gastrointestinal function in patients who are critically ill. Lancet Gastroenterol Hepatol. 2018;3(4):281-287. doi:10.1016/S24681253(18)30036-0

30. Blayney DW, Zhang Q, Feng J, et al. Efficacy of Plinabulin vs Pegfilgrastim for Prevention of Chemotherapy-Induced Neutropenia in Adults With Non-Small Cell Lung Cancer: a Phase 2 Randomized Clinical Trial. JAMA Oncol. 2020;6:11. doi:10.1001/jamaoncol.2020.4429
31. Aoyama T, Kawabe T, Fujikawa H, et al. Loss of Lean Body Mass as an Independent Risk Factor for Continuation of S-1 Adjuvant Chemotherapy for Gastric Cancer. Ann Surg Oncol. 2015;22 (8):2560-2566. doi:10.1245/s10434-014-4296-z

32. Migita K, Matsumoto S, Wakatsuki K, et al. A decrease in the prognostic nutritional index is associated with a worse long-term outcome in gastric cancer patients undergoing neoadjuvant chemotherapy. Surg Today. 2017;47(8):1018-1026. doi:10.1007/ s00595-017-1469-y

33. Awad S, Tan BH, Cui H, et al. Marked changes in body composition following neoadjuvant chemotherapy for oesophagogastric cancer. Clin nutr. 2012;31(1):74-77. doi:10.1016/j.clnu.2011.08.008

34. Tan BH, Brammer K, Randhawa N, et al. Sarcopenia is associated with toxicity in patients undergoing neo-adjuvant chemotherapy for oesophago-gastric cancer. Eur j Surg Oncol. 2015;41(3):333-338. doi:10.1016/j.ejso.2014.11.040

35. Ryan AM, Power DG, Daly L, Cushen SJ, Ní Bhuachalla E, Prado CM. Cancer-associated malnutrition, cachexia and sarcopenia: the skeleton in the hospital closet 40 years later. Proc Nutr Soc. 2016;75(2):199-211. doi:10.1017/S002966511500419X

36. Sano T, Sasako M, Yamamoto S, et al. Gastric cancer surgery: morbidity and mortality results from a prospective randomized controlled trial comparing D2 and extended para-aortic lymphadenectomy-Japan Clinical Oncology Group study 9501. J Clin Oncol. 2004;22(14):2767-2773. doi:10.1200/JCO.2004.10.184

\section{Publish your work in this journal}

Cancer Management and Research is an international, peer-reviewed open access journal focusing on cancer research and the optimal use of preventative and integrated treatment interventions to achieve improved outcomes, enhanced survival and quality of life for the cancer patient.
The manuscript management system is completely online and includes a very quick and fair peer-review system, which is all easy to use. Visit http://www.dovepress.com/testimonials.php to read real quotes from published authors. 